\title{
Battery Characterization and SOC/SOH Estimation
}

\author{
Saeid Habibi \\ Department of Mechanical Engineering \\ McMaster University, Canada
}

To optimize battery operation, it is important to monitor and estimate its State-of-Health ( $\mathrm{SOH}$ ) and State-of-Charge (SOC). These however cannot be directly measured, and need to be estimated. Model-based state and parameter estimation techniques are commonly used in SOC estimation, and are essential tools for information extraction. This presentation considers advanced testing strategies that could be used for characterization and modeling of batteries for SOC estimation. 